\title{
Designing green battery materials from Phenanthroline using Liquid Crystals
}

\author{
Amal A. Altalhi* and Ohood Z. Althaqafi \\ Chemistry department, College of science, Taif University, P.O. Box 1109, \\ Taif 21944, Saudi Arabia \\ *Corresponding author
}

A B S T R A C T

Keywords

Liquid crystals, Phenanthroline, Photogalvanic cell, Brij-30, Triton-X100 , Photocurrent, Photopotential, Diffusion coefficients

Article Info

Accepted:

12 December 2020 Available Online: 10 January 2021
Current lithium ion battery technologies suffer from challenges derived from the eco-toxicity, costliness, and energetic inefficiency of contemporary inorganic materials used in these devices. 1,1'diethylferrocene was studied which turned out to be successful as a potential half-cell to the battery. The ferroin complex was then looked at to complete the battery system which was also successful, so a test of the two systems as a battery system was carried out, with a voltage of $+0.240 \mathrm{~V}$ being observed, however the anti-dandruff shampoo was studied due to the zinc pyrithione this was studied successfully; cerium was then also studied due to its potential of giving rise to large voltages. Initially a low concentration of thallium was tested $(1 \mathrm{mM})$ where a voltage of $+832 \mathrm{mV}$ was observed, which was promising so $10 \mathrm{mM}$ and $50 \mathrm{mM}$ of the thallium were tested with the shampoo where voltages of $+1.306 \mathrm{~V}$ and $+1.503 \mathrm{~V}$ for each concentration respectively.

\section{Introduction}

Any form of renewable energy is regarded highly as the earth natural supply of fossil fuels is at dangerously low levels ${ }^{1}$, not only that the damage that is being done to the atmosphere by these fuels ${ }^{2}$, many different conditions have been tested with the ambition to finding an optimum photogalvanic cell ${ }^{3}$, we have seen that using well known dyes that being sensitive to light can especially when multiple dyes are used; there hugely improve not only the electrical output of the cell but its efficiency as well. The battery will consist of using lyotropic liquid crystals ${ }^{4,5}$ as a medium for the electrochemical process to occur, where the redox species is contained within the micelles of the liquid crystal and the electron transfer between the micelles. The lamella $^{6}$ and hexagonal ${ }^{7}$ phase will each be used to determine whether a certain phase induces a better potential difference. The experiments using iron sulphate ${ }^{7}$ and phenanthroline $^{8}$ to determine whether this combination would be effective as the second half to the battery system, the idea behind this 
system is that the iron (II) species $^{9}$ is coordinates to the phenanthroline to form $\mathrm{Fe}$ (phen) $)_{3}{ }^{2+}$ known as the ferroin complex. ${ }^{10}$ The iron species can be continuously oxidised and reduced from iron (II) to iron (III) and back to iron (II) which makes this an ideal substance to complete the battery.

\section{Materials and Methods}

Potassium chloride and hydrochloric acid solutions of $0.1 \mathrm{M}$ for each were used as the supporting electrolytes during these experiments. The potassium chloride was supplied from Fluka Chemicals with a purity of greater than $99.5 \%$ with the hydrochloric acid being supplied from Fischer Scientific with an initial concentration of $36 \% \mathrm{w} / \mathrm{v}$.

\section{Experimental procedures}

\section{Shampoo/ Zinc pyrithione and 2- Mercaptopyridine}

The Head and Shoulders shampoo had cyclic voltammetry carried out on, $0.1588 \mathrm{~g}, 100 \mathrm{mM}$ of zinc pyrithione and $0.06356 \mathrm{~g}, 100 \mathrm{mM}$ of 2-mercaptopyridine was dissolved in $5 \mathrm{~g}$ of Triton-X-100, where $5 \mathrm{~g}$ of $0.1 \mathrm{M}$ potassium chloride was added, this mixture was stirred and heated to $\approx 80^{\circ} \mathrm{C}$ for 1 hour. ${ }^{11}$ The mixture was then placed solely into the magnet with the horizontal magnetic field to cool; again voltammetry was carried out on the resulting sample.

\section{Instrumentation}

The machine used to carry out the cyclic voltammetry is a microAutolab Type III in connection with GPES software. Scanning electron microscopy was carried out using a Carl Zeiss SMT, Model: Evo 60, with an Energy Dispersive X-ray Spectrometer connected made by Oxford Instruements, Model: Inca Energy 350.

\section{Results and Discussion}

\section{Results from the Phenanthroline}

Again upon cooling cyclic voltammetry was carried out on each sample at room temperature which was measure to be $22^{\circ} \mathrm{C}$ the voltammetry was carried out over a range from $+0.6 \mathrm{~V}$ to $+1.2 \mathrm{~V}$ and back to $+0.6 \mathrm{~V}$ with a step potential of 0.00305 with graphs 1 and 2 showing the voltammograms of the samples in the vertical magnet filed followed by the horizontal magnet filed. From the voltammograms it is clear the reaction is reversible and the kinetics show this in graph 3: Both samples show linear relationship even at higher scan rates indicating the kinetics can keep up and maintain Nernst conditions. We can as described previously use the peak current to calculate the diffusion coefficient of each sample graph 4 gives the values for the gradient. Table 1 shows the values obtained for the diffusion coefficient of each sample where the concentration of the iron (II) phenanthroline complex is $3.234189 \mathrm{mM}$, the volume of the $\mathrm{H}_{1}$ phase is $9.0683 \mathrm{ml}$.

From the table it is clear the diffusion coefficients $^{12}$ remain the around the typical values as expected notably however is that the values for the oxidation and reduction of the sample from the vertical magnet filed have a constant diffusion coefficient. Having all this information in place allows a battery to potentially be made.

\section{Results from the Creation of the Battery System}

The battery was created by using two platinum electrodes, diagram 1 below shows what one half of the battery looks like:

The copper wire is connected to the platinum, with the other end can be connected up to an external circuit. Initially the 1,1'diethylferrocene ${ }^{13}$ solution was again reheated 
to melt the gel phase, the battery base is placed within the magneict filed, this was followed by the solution being poured onto the battery and left too cool within the magnetic field. This process was repeated again with the second half of the battery. Once the two halves had cooled they once was placed on top of the other to complete the battery. Using the voltammeter an initial reading of $+240 \mathrm{mV}$ was observed. However left overnight and the battery had selfdischarged ${ }^{14}$ and the two mixtures had mixed, this is meaning the rate of diffusion between the two phases was really quick, indicating a different method was needed to generate the battery, ordinary shampoo was used due to the main component zinc pyrithione where the zinc can reduce to zinc (0) and back to zinc (II)

\section{Shampoo results}

Based on reducing zinc to its metallic form using cyclic voltammetry a stripping peak should be seen as the zinc metal ${ }^{15}$ is deposited onto the electrode surface, graph 5 shows the voltammograms obtained:

The voltammetry was again carried out over three scans with the first scan at each scan rate being taken for analysis, the scans were done over a range from $0 \mathrm{~V}$ to $-1.7 \mathrm{~V}$ and back to $0 \mathrm{~V}$ with a step potential of $0.00305 \mathrm{~V}$.From the voltammograms it is clear that there is a stripping peak where the zinc metals is stripped from the electrode and back into solution the kinetics also indicate the potential of this system.

Table.1 Diffusion coefficients within each sample

\begin{tabular}{|c|c|c|}
\hline $\operatorname{Gradient}\left(A \mathbf{V}^{-1}\right)$ & Magnet & Diffusion Coefficient $\left(\mathrm{cm}^{2} \mathrm{~s}^{-1}\right)$ \\
\hline $2.15476 \times 10^{-5}$ & the vertical magnet Oxidation & $1.22 \times 10^{-7}$ \\
\hline $1.56809 \times 10^{-5}$ & $\begin{array}{c}\text { the horizontal magnet } \\
\text { Oxidation }\end{array}$ & $6.45 \times 10^{-8}$ \\
\hline$-2.17749 \times 10^{-5}$ & the vertical magnet Reduction & $1.24 \times 10^{-7}$ \\
\hline$-1.40562 \times 10^{-5}$ & $\begin{array}{l}\text { the horizontal magnet } \\
\text { Reduction }\end{array}$ & $5.19 \times 10^{-8}$ \\
\hline
\end{tabular}

Graph.1 Cyclic voltammograms of the sample in the vertical magnet filed

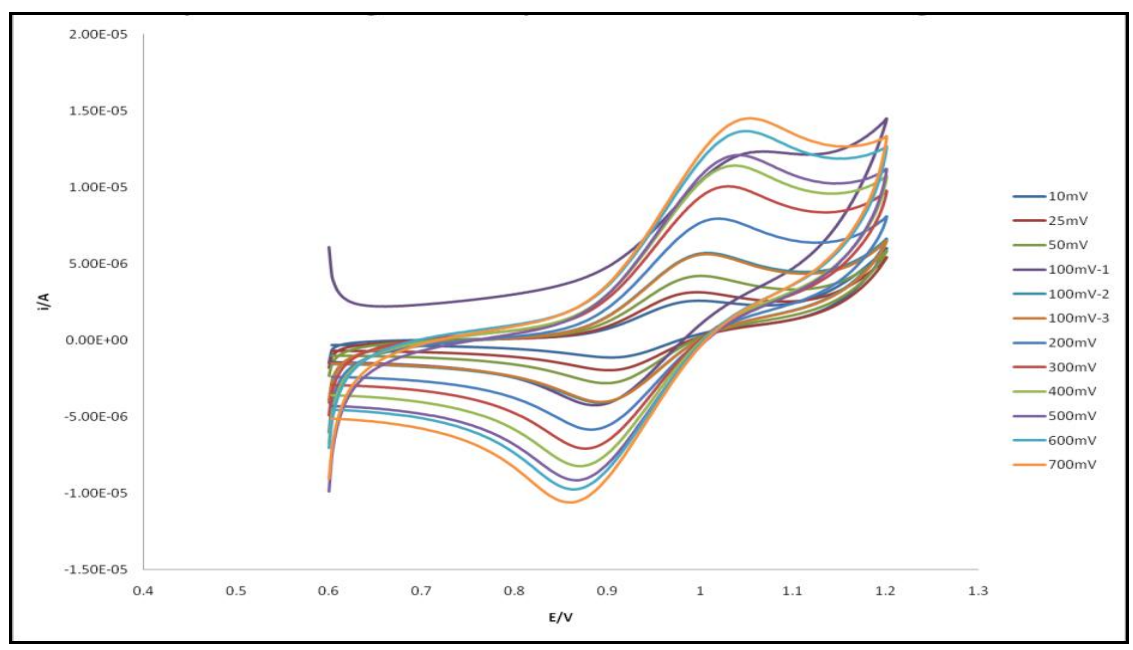


Graph.2 Cyclic voltammograms of the sample in the horizontal magnet

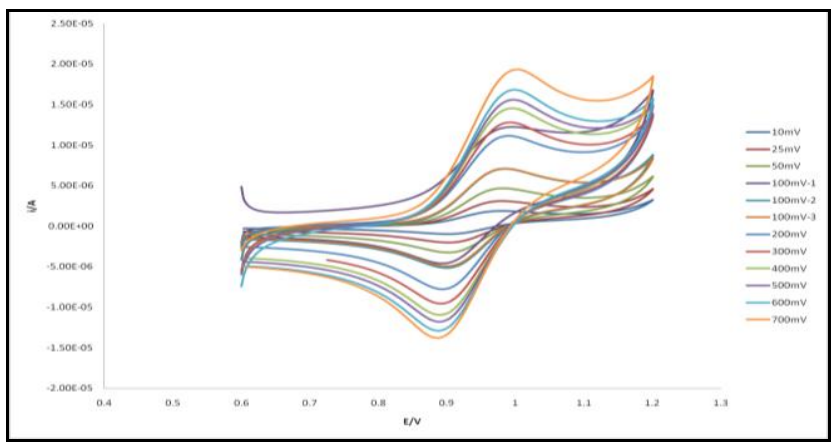

Graph.3 Peak potential against $\log _{10}$ of scan rate

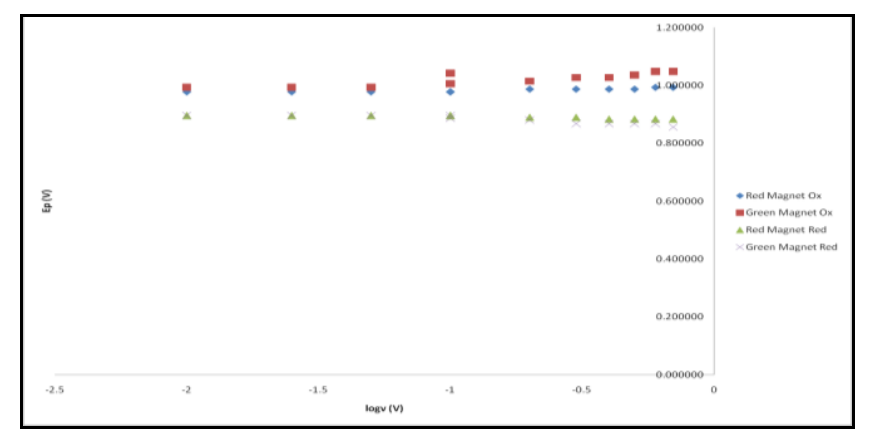

Graph.4 Peak current against square root of scan rate

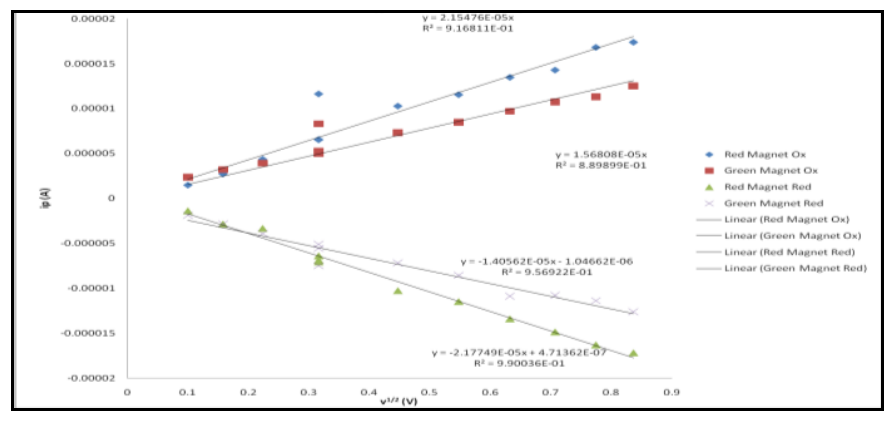

Graph.5 Voltammograms of the shampoo

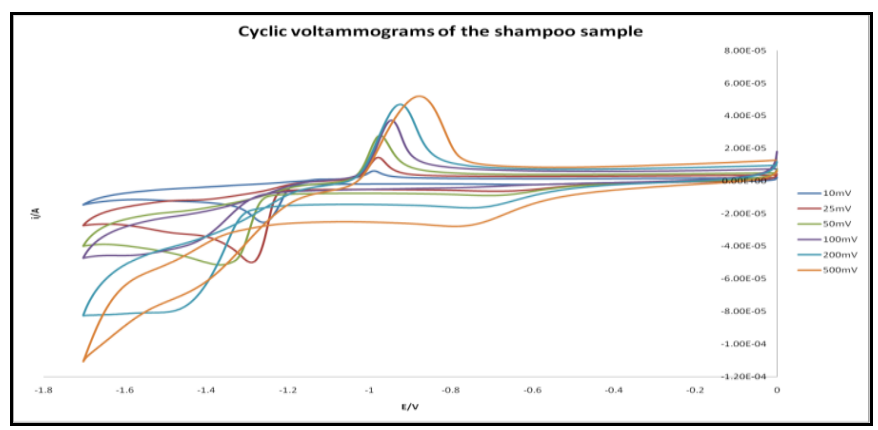


Graph.7 Voltammograms of the pure zinc pyrithione

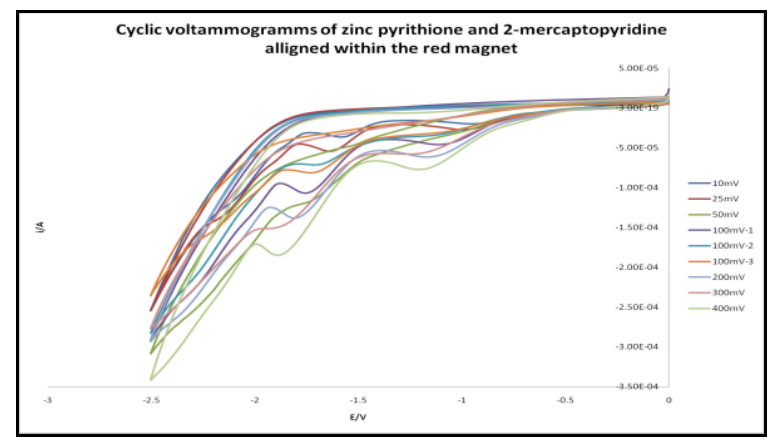

Graph.8 X-ray diffraction results from the electrode

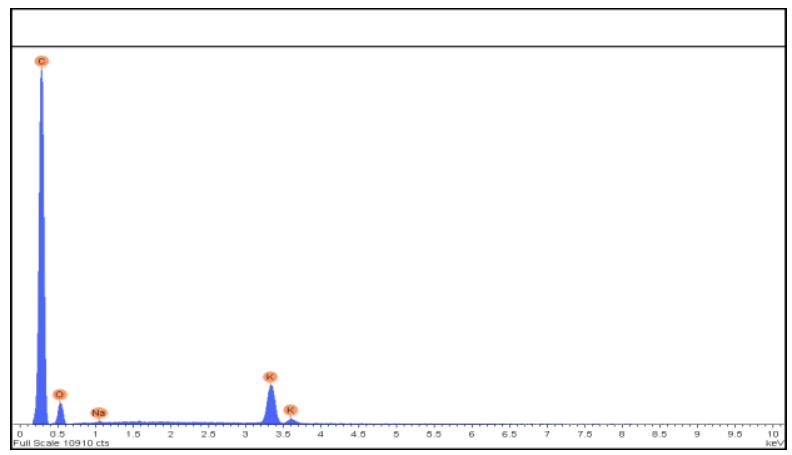

Graph.9 Where zinc would be expected to be seen during analysis

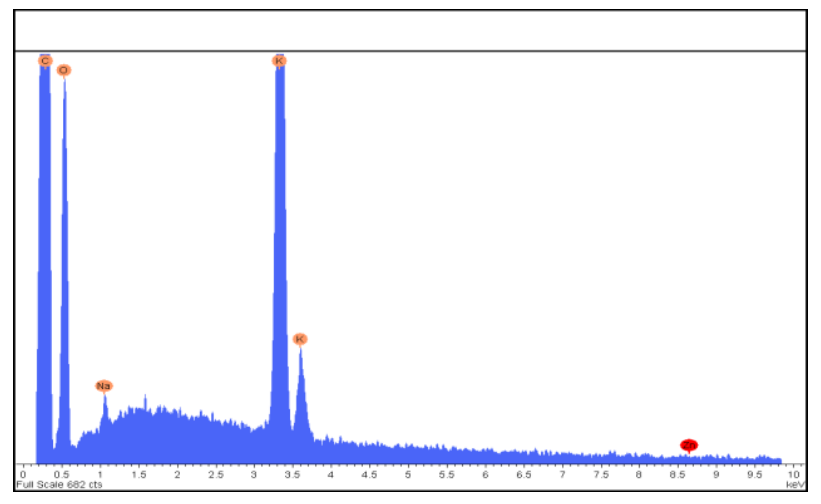

Diagram.1 Design of the battery used 


\section{Diagram.2 Scanning Electron Image of the electrode}

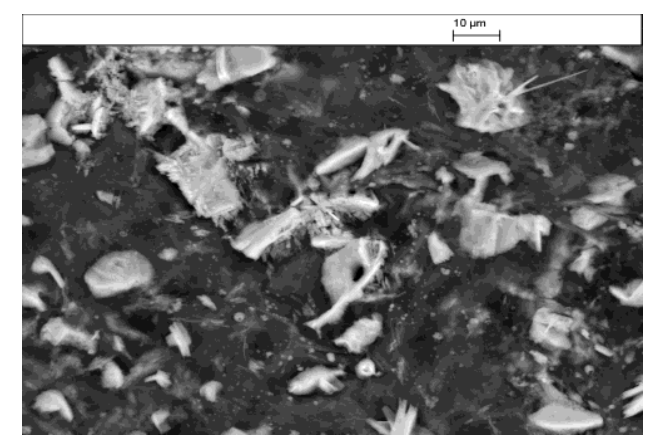

\section{Zinc Pyrithione/ 2-Mercaptopyridine results}

$159.5 \mathrm{mg}(100 \mathrm{mM})$ of the zinc pyrithione and $64.2(100 \mathrm{mM})$ of the 2-mercaptopyridine was dissolved in $5.0491 \mathrm{~g}$ of $0.1 \mathrm{M} \mathrm{KCl}$ to that $5.0198 \mathrm{~g}$ of the Triton-X-100 was added. ${ }^{16}$ The sample was only cooled within the red magnet and again voltammetry was carried out giving results shown in graph 7 below.

It is clear initially there is no stripping peaks meaning in this case no zinc metal is deposited onto the electrode surface, there is the reduction of zinc around $-1.75 \mathrm{~V}$ as expected but no peak to suggest it is reoxidised the voltammetry was carried out from $0 \mathrm{~V}$ to $-2.5 \mathrm{~V}$ back to $0 \mathrm{~V}$ with a step potential of $0.00305 \mathrm{~V}$.Diagram 2 shows an elemental contrast from a scan electron microscope indicating only potassium was picked up, graphs 8 and 9 show the x-ray diffraction information collected and then how the graph should look if zinc had been present:

Elemental contrast was used as the detector where elements heavier than carbon are shown in brighter colours, based on what is seen in graph 8 these white parts are potassium.

As expected there is a strong carbon peak as a carbon based electrode was used, A small amount of sodium was observed but not a significant amount, with a prominent peak for potassium, but no zinc was detected, meaning what is seen in diagram 2 is that of potassium and not zinc.

This a more enhanced version of graph 8 however this includes zinc in bright red, which means that no zinc was detected but if there had been zinc then this is where a peak would have been observed. From the voltammograms discussed previously there is no correlation between all the peaks meaning this is irreversible, this means there must be some other compound within the shampoo to aid the reduction of the zinc and allow it to form back into solution.

In conclusion, the initial aims set out have been met, as a successful battery system had been generated, after initially studying many different potential half cells a thallium based and a shampoo based cell. Obtaining an initial voltage of $+1.503 \mathrm{~V}$ that was maintained over a sustained period of time. In the future the battery will become adaptable to work via solar energy conversion, with a look at less toxic metals due to thallium's high toxicity.

\section{Acknowledgement}

We would like to thank Taif University Researcher supporting project number (TURP-2020/243), Taif University, Taif, Saudi Arabia. 


\section{References}

1. K.M. Gangotri and M.K. Bhimwal, Solar Energy, 2010, 84, 1294

2. P. Atkins and J.D. Paula, Elements of PhysChem, 2005, 4, 292

3. T. Zawodzinski, S. Minteer and G.Brisard, Interface, 2006, 15, 62

4. J. Daintith, Oxford Dictioary of Chemistry, 2004, 5, 439

5. C.H. Hamann, A. Hamnett and W. Vielstich, Electrochemistry, 1998, 1, 338

6. C.H. Hamann, A. Hamnett and W. Vielstich, Electrochemistry, 1998, 1, 212

7. J. Wadhawan, 06729 Hot topics Lecture course, 2010/2011, 41

8. D.B. Hibbert, Intro to Electrochemistry, 1993, 137

9. M. Grätzel, Journal of Photochemistry and Photobiology, 2003, 4, 145

10. K.R. Genwa, A. Kumar and A. Sonel, Applied Energy, 2009, 86, 1431

11. K.R. Genwa and A. Chouhan, Solar energy, 2006, 80, 1213

12. B.B. Bhowmik, R. Choudhary and K.K. Rohtgi-Mukherji, Indian Journal of Chemistry, 1987, 26A, 95

13. C. Lal, Journal of Power Sources, 2007, 164, 926

14. K.M. Gangotri, Journal of Photochemistry and Photobiology A: Chemistry, 2001, 14, 175

15. K.M. Gangotri and V. Indora, Solar Energy, 2010, 84, 271

16. S. Pokhrel and K.S. Nagaraja, Solar Energy Materials \& Solar Cells, 2009, 93, 244

\section{How to cite this article:}

Amal A. Altalhi and Ohood Z. Althaqafi. 2021. Designing green battery materials from Phenanthroline using Liquid Crystals. Int.J.Curr.Microbiol.App.Sci. 10(01): 920-926. doi: https://doi.org/10.20546/ijcmas.2021.1001.110 